Originally published as:

Mugisha, L., Leendertz, F.H., Opuda-Asibo, J., Olobo, J.O., Ehlers, B.

A novel herpesvirus in the sanctuary chimpanzees on Ngamba Island in Uganda (2010) Journal of Medical Primatology, 39 (1), pp. 71-76.

DOI: 10.1111/j.1600-0684.2009.00396.x

The definitive version is available at http://www3.interscience.wiley.com 


\title{
A novel herpesvirus in the sanctuary chimpanzees on Ngamba Island in Uganda
}

\author{
L. Mugisha ${ }^{1,2}$, F.H. Leendertz ${ }^{2}$, J. Opuda-Asibo ${ }^{3}$, J.O. Olobo3 \& B. Ehlers ${ }^{4}$ \\ 1 Chimpanzee Sanctuary \& Wildlife Conservation Trust (CSWCT), Entebbe, Uganda \\ 2 Research group "Emerging Zoonoses”, Robert Koch-Institute, Berlin, Germany \\ 3 Makerere University, Department of Wildlife and Animal Resources Management (WARM), Kampala, Uganda \\ 4 Research group "Molecular Genetics and Epidemiology of Herpesviruses", Robert Koch-Institute, Berlin, Germany
}

\begin{abstract}
Background: Recent studies in non-human primates have led to the discovery of novel primate herpesviruses. In order to get more information on herpesvirus infections in apes, we studied wild born captive chimpanzees.

Methods: Chimpanzees of the Ngamba island sanctuary, Uganda, were analyzed with pan-herpes polymerase chain reaction (PCR) targeting the herpesvirus DNA polymerase gene and the glycoprotein B gene. The obtained sequences were connected by long-distance PCR, and analyzed phylogenetically.

Results: Twenty-one of 40 individuals were infected with members of the Gammaherpesvirinae, two of them with a novel member of this subfamily. Phylogenetically, the novel virus fell into a clade of primate rhadinoviruses and the Kaposi sarcoma herpesvirus (human herpesvirus 8), representing a third distinct rhadinovirus in chimpanzees.

Conclusion: Non-human primates harbor several herpesviruses many of which are still unknown. This has implications to management of primates in sanctuaries requiring continuous updates on the management protocols to deal with potential occupational pathogens.
\end{abstract}

\section{Introduction}

Little is known about the spectrum of infectious agents carried by wild non-human primates. However, such baseline data would be helpful to identify potential zoonotic threats and to better understand players involved in diseases threatening the health of nonhuman primates $[11,20]$. Herpesviruses may play an important role in multifactorial disease progressions, and despite various discoveries of novel herpesviruses in wild great apes and other primates [8, 18, 20], the spectrum of herpesviruses is far from being described.

The members of the family Herpesviridae have been previously grouped into three subfamilies, designated Alphaherpesvirinae, Betaherpesvirinae and Gammaherpesvirinae [28], and phylogenetic lineages in these subfamilies were identified [22-25]. In studies amplifying unknown herpes virus sequences from the DANN polymerase (DPOL) gene or - more recently - from two conserved genes by a bigenic polymerase chain reaction (PCR) approach, novel herpesviruses were continuously discovered. For example, 14 novel gammaherpesviruses (GHV) were discovered in eight different host species from six mammalian orders [8], and 10 novel betaherpesviruses were identified in great apes [19]. These discoveries were based on sequences encompassing two or more conserved genes, and determined from long-distance (LD)-PCR products [8, 9, 19, 27].

Herpesviruses are widespread in vertebrate species, sharing several moderately to well-conserved genes, as determined from amino acid identity comparisons (e.g. DNA polymerase and glycoprotein B). Within the GHV, Epstein-Barr virus (EBV) and Kaposi's sarcoma-associated herpesvirus (KSHV), classified as Human herpesvirus 4 (HHV-4) and Human herpesvirus 8 (HHV-8), are the human members of the Lymphocryptovirus genus and the Rhadinovirus genus respectively. Both of these viruses play a critical role in human multistep carcinogenesis, especially in immunodeficient patients, leading to Burkitt's lymphoma [21] and Kaposi's sarcoma [5, 31] respectively. GHV of the genus Rhadinovirus have also been detected in several mammalian species including New World monkeys (squirrel monkey, spider monkey) [1, 2] and Old World monkeys (several macaque species, African green monkey, mandrill) $[3,7,13,15,29,32]$. Comparison and phylogenetic analyses of available sequences support the existence of two distinct lineages among the Old World monkey rhadinoviruses, called RV1 and RV2 for the Rhadinovirus lineages 1 and 2 [4, 13, 15, 30]. KSHV belongs to the RV1 lineage, whereas no human virus has yet been discovered in the RV2 lineage. 
Previous studies on African great apes from Cameroon and Gabon have shown that they harbor different rhadinoviruses closely related to KSHV. By serology and PCR, common chimpanzees were determined to be infected with rhadinoviruses of the RV1 and the RV2 lineage being termed Pan Rhadino-herpesvirus 1a, 1b, 2 (PanRHV1a, PanRHV1b, PanRHV2) [16, 17]. Using a bigenic LD-PCR approach, rhadinoviruses very similar to PanRHV1a and PanRHV2 were found in chimpanzees from Ivory Coast (PtroRHV-1 and PtroRHV-2) [27]. These studies demonstrate that wild animals, especially primates, harbor many unknown viruses, and further search will continue to reveal novel pathogens.

In this study, we analyzed wild born captive chimpanzees residing at Ngamba Sanctuary in Uganda to gain more insights into the herpesviruses harbored by this community. The results would fit into improvements of management protocols including employee health programs and future plans for reintroductions.

\section{Materials and methods}

The study was conducted on 40 orphan chimpanzees (Pan troglodytes schweinfurthii) in semi-captive management at Ngamba Island Chimpanzee Sanctuary, Uganda. The total group consists of 23 females and 19 males with age group categorized as follows: 4 infants (1-5 years), 6 juveniles (6-8 years), 12 subadults (9-11 years) and adults (12 years and more). These chimpanzees were brought to the sanctuary at different times after being rescued from illegal traders and poachers mainly from Uganda and Democratic Republic of Congo since 1998 when the sanctuary started.

Blood was collected from 40 chimpanzees under general anesthesia during our annual medical health checks in February 2007 . Whole blood $(10 \mathrm{ml})$ was taken by inguinal venipuncture using CPT Vacutainer tubes. Leucocytes/cells from fresh whole blood collected in CPT tubes were processed using a cell preparation procedure within 12 hours. The processed cells were stored in cryotubes under liquid nitrogen $\left(-196^{\circ} \mathrm{C}\right)$ and stored at Uganda Virus Research Institute till transported on dry ice to Robert Koch Institute for analysis.

The research was approved by Uganda Wildlife Authority and Uganda National Council of Science and Technology. In addition to international requirements, the local and regional requirements of managing chimpanzees in sanctuaries established by International Primatological Society and Pan African Sanctuaries Alliance were followed as part of routine management of chimpanzees.

\section{DNA extraction, amplification, purification, cloning and sequencing}

DNA was extracted from buffy coat of the whole blood cells of 40 chimpanzees using QIAamp DANN Blood Min Kit (QIAGEN®, QIAamp®, Hilden, Germany) according to manufacturer's instructions.

Panherpesvirus consensus PCR for the amplification of 160-181 bp (excluding primer-binding sites) of the DPOL gene was carried out as described previously [6]. Samples that did not yield an amplification product were rerun under more relaxed conditions; i.e. the ramp time between the annealing step and the extension step was prolonged 50 -fold, and the final concentration of the polymerase was increased twofold.

For the amplification of the gB genes of as yet unknown GHVs, two degenerate, deoxyinosinecontaining primer sets ( $\mathrm{GH} 1$ and $\mathrm{GH} 2$ ) were used [8].

Nested LD-PCR was performed with the TaKaRa-Ex PCR system according to the instructions of the manufacturer (Takara Bio Inc., Otsu, Japan) by using virus-specific primers (not listed) as described previously $[8,12]$. PCR products were purified by using the PCR purification kit (QIAGEN) and directly sequenced with the Big Dye terminator cycle sequencing kit (Applied Biosystems, Warrington, UK) in a 377 DNAautomated sequencer (Applied Biosystems).

\section{Multiple sequence alignment and phylogenetic analysis}

Analysis was carried out at the level of encoded amino acid sequences. Sets of partial amino acid sequences from DPOL and gB were aligned using clustalw [33]. Positions in alignments that had 
missing characters in any sequence were removed before the alignment was used for phylogenetic analyses with the neighbor-joining method as performed previously [27].

\section{Nucleotide sequence accession numbers}

The following DPOL and gB sequences of known primate herpesviruses were used for comparative purposes: EBV = HHV-4, GenBank accession number (acc.-no.) NC_009334; Pan troglodytes lymphocryptovirus (PtroLCV-1), herpesvirus pan = Panine herpesvirus 1 (PnHV-1), acc.-no. AF534226; Rhesus lymphocryptovirus (RhLCV) = Macacine herpesvirus 4 (McHV-4), acc.-no. NC_006146; Marmoset lymphocryptovirus (MaLCV) = Callitrichine herpesvirus 3 (CalHV-3), acc.-no. NC_004367; KSHV = HHV-8, acc.-no. NC_003409; Rhesus rhadinovirus (RRV) = Macacine herpesvirus 5 (McHV-5), acc.-no. NC_003401; Pan troglodytes rhadinovirus 1 (PtroRHV-1), acc.-no. AY138585; Pan troglodytes rhadinovirus 2 (PtroRHV2), acc.-no. AY138585; Macaca fascicularis rhadinovirus 1 (MfasRHV-1), acc.-no. AY138583; Macaca fascicularis rhadinovirus 2 (MfasRHV2), acc.-no. EU085377; Retroperitoneal fibromatosis-associated herpesvirus (RFHV), acc.-no. AF204166; Herpesvirus Saimiri (HVS) = Saimiriine herpesvirus 2 (SaHV-2), acc.-no. NC_001350; Chimpanzee cytomegalovirus $(\mathrm{CCMV})=$ Panine herpesvirus 2, acc.-no. AF480884. The nucleotide sequence of PtroRHV-3 was deposited in GenBank under the acc.-no. GQ995451).

\section{Results and discussion}

Twenty-one of 40 samples from chimpanzees (Pan troglodytes schweinfurthii) analyzed with panherpes DPOL PCR were tested positive for GHV. The sequences from five samples originated from PtroRHV- 1 (acc.-no. AY138585) [27]. Fourteen samples were positive for the lymphocryptovirus PtroLCV-1 (acc.-no. AF534226) [10]. Two cases appeared to harbor a novel gammaherpesvirus, which was tentatively named Pan troglodytes rhadinovirus 3 (PtroRHV-3). All results are listed Table 1.

With degenerate primers targeting the gB gene, a 453-bp sequence was amplified from one of the PtroRHV-3-positive samples. From the partial gB and DPOL sequences of PtroRHV-3, specific nested primers were deduced and used in LD-PCR. The 3.3-kb product was sequenced and a final contiguous PtroRHV3 sequence was compiled spanning 3446 bp.

In a pair-wise nucleic acid sequence comparison, the 3.4-kbp sequence of PtroRHV-3 was found to be $69 \%$ identical to PtroRHV-1. The 3 \$end of the PtroRHV-3 sequence $(0.4 \mathrm{~kb})$ was $99 \%$ identical to a rhadinovirus (PanRHV1b) found in Pan troglodytes troglodytes [17]. No close matches were found in GenBank for the $5 \$$-part (3 kbp) of the PtroRHV-3 sequence, spanning a part of the DPOL and the gB gene. As the PtroRHV-3 sequence was detected in Pan troglodytes schweinfurthii from Uganda and the Democratic Republic of Congo, it was regarded as originating from a hitherto unknown P. t. schweinfurthii RHV, closely related to P. t. troglodytes RHV1b.

From the partial gB and DPOL open reading frames (1020 and 2376 bp respectively), amino acid sequences were deduced, concatenated and aligned with corresponding aa primate GHV sequences from GenBank. Phylogenetic tree analysis was performed as described previously [27], and aa sequences of the chimpanzee cytomegalovirus (GenBank acc.-no. AF480884) were used for rooting. In the tree, the novel virus clustered more closely with the human rhadinovirus (KSHV/ HHV-8) and with PtroRHV-1 than with PtroRHV2 (Fig. 1).

This study confirms that there are three distinct rhadinoviruses within the Gammaherpesvirinae of chimpanzees [16]. Although the human rhadinovirus HHV-8 is closely related to PtroRHV-1 (and PanRHV1a), no human rhadinoviruses with close similarity to PtroRHV-2 or PtroRHV-3 are known. Nevertheless, they could exist, as herpesviruses co-evolve with their hosts.

The human gammaherpesviruses EBV and KSHV pose significant health risks worldwide, including the induction of cancer and lymphoproliferative diseases. They pose a risk of serious disease especially to those lacking a competent immune system, such as neonates, transplant patients and people suffering from AIDS (reviewed in Ref. [26]). Although acute gammaherpesvirus infection is rapidly cleared by a strong immune response, the establishment of latent infection in hematopoietic 
cells allows these viruses to successfully evade the host immune response and maintain lifelong infection. To date, the pathogenesis and immunity of gammaherpesvirus infections are still not well understood and therefore convincing strategies to prevent long-term latency have not been realized [35].

It has been demonstrated in studies with mice that the level of latent infection with a gammaherpesvirus is independent of the infectious dose over at least a 104-fold range, regardless of whether inoculation occurs via a systemic or a mucosal route [34]. Host factors like a compromised immune system have been implicated in the initiation of infection leading to substantial acute phase replication and subsequent establishment of a maximal level of latency. In addition, it is not known whether the gammaherpesviruses found in great apes present the same way as their human counterparts both in establishment of infection and pathogenicity. For example, it has now been shown that chimpanzees harboring simian immunodeficiency virus have a higher likelihood of dying and lower reproductive rate [14]. Most likely, the underlying pathological processes are multifactorial, and herpesviruses may play an important role. Therefore, detailed analysis in long-term studies is needed.

Finally, not only co-evolvement with the host but also horizontal transmission appears to be an important component of beta- and gammaherpesvirus evolution [8, 19; B. Ehlers and D. McGeoch, unpublished data]. These circumstances and uncertainties present challenges in the management of occupational hazards in captive ape facilities (sanctuaries and zoos) where there is close interaction of employees with the apes.

\section{Acknowledgments}

This research was carried out within the frame of the network 'Great Ape Health Monitoring Unit (GAHMU)'. The analyses were supported by Robert Koch-Institute, Berlin; Brian Hare, Max Planck Institute for Evolutionary Anthropology, Leipzig and Kim Hammond, Falls Road Hospital, Baltimore, Maryland through MGVP. The authors thank Chimpanzee Sanctuary \& Wildlife Conservation Trust, Uganda Wildlife Authority, Uganda National Council of Science and Technology and the CITES offices of Uganda and Germany for permission and permits to carry out this research. The authors also thank Nezlisah Yasmum for excellent technical assistance.

\section{References}

1 Albrecht JC: Primary structure of the Herpesvirus ateles genome. J Virol 2000; 74:1033-7. 2 Albrecht JC, Fleckenstein B: Structural organization of the conserved gene block of Herpesvirus saimiri coding for DNA polymerase, glycoprotein B, and major DANN binding protein. Virol 1990; 174:533-42.

3 Auerbach MR, Czajak SC, Johnson WE, Desrosiers RC, Alexander L: Species specificity of macaque Rhadinovirus glycoprotein B sequences. J Virol 2000; 74:584-90.

4 Bosch ML, Strand KB, Rose TM: Gammaherpesvirus sequence comparisons. J Virol 1998; 72:8458-9.

5 Chang Y, Cesarman E, Pessin MS, Lee F, Culpepper J, Knowles DM, Moore PS: Identification of herpesviruslike DNA sequences in AIDS-associated Kaposi's sarcoma. Science 1994; 266:1865-9. 6 Chmielewicz B, Goltz M, Ehlers B: Detection and multigenic characterization of a novel gammaherpesvirus in goats. Virus Res 2001; 75:87-94.

7 Desrosiers RC, Sasseville VG, Czajak SC, Zhang X, Mansfield KG, Kaur A, Johnson RP, Lackner AA, Jung JU: A herpesvirus of rhesus monkeys related to the human Kaposi's sarcoma-associated herpesvirus. J Virol 1997; 71:9764-9.

8 Ehlers B, Dural G, Yasmum N, Lembo T, de Thoisy B, Ryser-Degiorgis MP, Ulrich RG, McGeoch DJ: Novel mammalian herpesviruses and lineages within the Gammaherpesvirinae: cospeciation and interspecies transfer. J Virol 2008; 82:3509-16.

9 Ehlers B, Kuechler J, Yasmum N, Dural G, Voigt S, Schmidt-Chanasit J, Jaekel T, Matuschka RF, Richter D, Essbauer S, Hughes DJ, Summers C, Bennett M, Stewart JP, Ulrich RG: Identification of novel rodent herpesviruses including the first gammaherpesvirus of Mus musculus. J Virol 2007; 81:8091-100. 
10 Ehlers B, Ochs A, Leendertz F, Goltz M, Boesch C, Mätz-Rensing K: Novel simian homologues of Epstein- Barr virus. J Virol 2003; 77:10695-9.

11 Gillespie TR, Nunn CL, Leendertz FH: Integrative approaches to the study of primate infectious disease: implications for biodiversity conservation and global health. Am J Phys Anthropol Suppl 2009; 47:53-69.

12 Goltz M, Ericsson T, Patience C, Huang CA, Noack S, Sachs DH, Ehlers B: Sequence analysis of the genome of porcine lymphotropic herpesvirus 1 and gene expression during post-transplant lymphoproliferative disease of pigs. Virol 2002; 294:383-93.

13 Greensill J, Sheldon JA, Renwick NM, Beer BE, Norley S, Goudsmit J, Schulz TF: Two distinct gamma-2 herpesviruses in African green monkeys: a second gamma- 2herpesvirus 8 in Africa: both $B$ and $A 5 \mathrm{~K} 1$ genotypes, as well as the $\mathrm{M}$ and $\mathrm{P}$ genotypes of K14.1/K15 loci, are frequent and widespread. Virol 2000; 278:60-74.

14 Keele BF, Jones JH, Terio KA, Estes JD, Rudicell RS, Wilson ML, Li Y, Learn GH, Beasley TB, Schumacher- Stankey J, Wroblewski E, Mosser A, Raphael J, Kamenya S, Lonsdorf EV, Travis DA, Mlengeya T, Kinsel MJ, Else JG, Silvestri G, Goodall J, Sharp PM, Shaw GA, Pusey AE, Hahn BH: Increased mortality and AIDS-like immunopathology in wild chimpanzees infected with SIVcpz. Nature 2009; 460:515-9.

15 Lacoste V, Mauclere P, Dubreuil G, Lewis J, Georges-Courbot MC, Gessain A: KSHV-like herpesviruses in chimps and gorillas. Nature 2000; 407:151-2.

16 Lacoste V, Mauclere P, Dubreuil G, Lewis J, Georges- Courbot MC, Gessain A: A novel c2herpesvirus of the rhadinovirus 2 lineage in chimpanzees. Genome Res 2001; 11:1511-9.

17 Lacoste V, Mauclere P, Dubreuil G, Lewis J, Georges-Courbot MC, Rigoulet J, Petit T, Gessain A: Simian homologues of human gamma-2 and betaherpesviruses in mandrill and drill monkeys. J Virol 2000; 74:11993-9.

18 Lacoste V, Verschoor EJ, Nerrienet E, Gessain A: A novel homologue of Human herpesvirus 6 in chimpanzees. J Gen Virol 2005; 86:2135-40.

19 Leendertz FH, Deckers M, Schempp W, Lankester F, Boesch C, Mugisha L, Dolan A, Duncan DJ, McGeoch J, Ehlers B: Novel cytomegaloviruses in free-ranging and captive great apes: phylogenetic evidence for bidirectional horizontal transmission. J Gen Virol 2009; 90:2386-94.

20 Leendertz FH, Pauli G, Maetz-Rensing K, Boardman W, Nunn C, Ellerbrok H, Jensen SA, Junglen $\mathrm{S}$, Boesch C: Pathogens as drivers of population declines: the importance of systematic monitoring in great apes and other threatened mammals. Biol Conserv 2006; 131:325-37.

21 Magrath I, Judde J: Epstein-Barr virus and neoplasia. In: Molecular biology of cancer. Bertino (ed.). NewYork: Academic Press, 1996; 642-51. 22 McGeoch DJ: Molecular evolution of the c-

Herpesvirinae. Philos Trans R Soc Lond 2001; 356:421-35.

23 McGeoch DJ, Dolan A, Ralph AC: Toward a comprehensive phylogeny for mammalian and avian herpesviruses. J Virol 2000; 74:10401-6.

24 McGeoch DJ, Gatherer D, Dolan A: On phylogenetic relationships among major lineages of the Gammaherpesvirinae. J Gen Virol 2005; 86:307-16.

25 McGeoch DJ, Rixon FJ, Davison AJ: Topics in herpesvirus genomics and evolution. Virus Res 2006; 117:90-104.

26 Pass RF: Cytomegalovirus. In: Fields Virology, 4th edn, Vol. 2. Knipe \& Howley (eds). Philadelphia, PA: Lippincott Williams \& Wilkins, 2001; 2675-705.

27 Prepens S, Kreuzer KA, Leendertz F, Nitsche A, Ehlers B: Discovery of herpesviruses in multiinfected primates using locked nucleic acids (LNA) and a bigenic PCR approach. Virology J 2007; 4:84.

28 Roizman B, Desrosiers RC, Fleckenstein B, Lopez C, Minson AC, Studdert MJ: The family

Herpesviridae: an update. The Herpesvirus Study Group of the International Committee on Taxonomy of Viruses. Arch Virol 1992; 123:425-49.

29 Rose TM, Strand KB, Schultz ER, Schaefer G, Rankin GW Jr, Thouless ME, Tsai CC, Bosch ML:

Identification of two homologs of the Kaposi's sarcoma-associated herpesvirus (human herpesvirus 8) in retroperitoneal fibromatosis of different macaque species. J Virol 1997; 71:4138-44.

30 Schultz ER, RankinGWJr, Blanc MP, Raden BW, Tsai CC, Rose TM: Characterization of two

divergent lineages of macaque rhadinoviruses related to Kaposi's sarcoma-associated herpesvirus. $\mathrm{J}$. Viro 2000; 74:4919-28.

31 Schulz TF: Kaposi's sarcoma-associated herpesvirus (human herpesvirus-8). J Gen Virol 1998; 79:1573-91.

32 Strand K, Harper E, Thormahlen S, Thouless ME, Tsai C, Rose T, Bosch ML: Two distinct lineages of macaque gamma herpesviruses related to the Kaposi's sarcoma associated herpesvirus. J Clin Virol 2000; 16:253-69. 
33 Thompson JD, Higgins DG, Gibson TJ: CLUSTAL W: improving the sensitivity of progressive multiple sequence alignment through sequence weighting, position-specific gap penalties and weight matrix choice. Nucleic Acids Res 1994; 22:4673-80.

34 Tibbetts SA, Loh J, van Berkel V, McClellan JS, Jacoby MA, Kapadia SB, Speck SH, Virgin IV HW: Establishment and maintenance of gammaherpesvirus latency are independent of infective dose and route of infection. J Virol 2003; 77:7696-701.

35 Tibbetts SA, McClellan JS, Gangappa S, Speck SH, Virgin IV HW: Effective vaccination against long-term gammaherpesvirus latency. J. Virology 2003; 77:2522-9.

\section{Figures and Tables}

Table 1 Chimpanzee biodata and gammaherpesviruses detected

\begin{tabular}{|c|c|c|c|c|c|c|}
\hline \multirow{2}{*}{\multicolumn{4}{|c|}{ Chimpanzee biodata }} & \multicolumn{3}{|l|}{ Gammaherpesvininae } \\
\hline & & & & \multirow{2}{*}{$\frac{\text { Lymphocryptovirus }}{\text { PtroLCV-1 }}$} & \multicolumn{2}{|c|}{ Rhadinovirus } \\
\hline Name of chimpanzee & Sex & Age & Origin & & PtroRHV-1 & PtroRHV-3 \\
\hline Kidogo & $\mathrm{F}$ & 23 & DRC & - & - & - \\
\hline Sophie & $\mathrm{F}$ & 21 & DRC & + & - & - \\
\hline Katie & $\mathrm{F}$ & 20 & DRC & + & - & - \\
\hline Ikuru & $\mathrm{F}$ & 12 & DRC & + & - & - \\
\hline Billi & $\mathrm{F}$ & 10 & DRC & + & - & - \\
\hline Mukisa & $\mathrm{F}$ & 9 & DRC & - & - & + \\
\hline Ndyakira & $\mathrm{F}$ & 8 & DRC & - & - & - \\
\hline Nakuu & $\mathrm{F}$ & 6 & DRC & + & - & - \\
\hline Masiko & $M$ & 23 & DRC & - & - & - \\
\hline Sunday & M & 20 & DRC & + & - & - \\
\hline Robbie & M & 21 & DRC & - & - & - \\
\hline Mawa & $M$ & 11 & DRC & - & - & - \\
\hline Kalema & $M$ & 11 & DRC & - & - & - \\
\hline Umutama & $\mathrm{M}$ & 11 & DRC & - & - & - \\
\hline Umugezi & M & 10 & DRC & + & - & - \\
\hline Baluku & $M$ & 9 & DRC & + & - & - \\
\hline Asega & M & 9 & DRC & - & - & - \\
\hline Kisembo & $M$ & 8 & DRC & + & - & - \\
\hline Indi & $M$ & 8 & DRC & + & - & - \\
\hline Okech & M & 8 & DRC & - & + & - \\
\hline Rambo & M & 3 & DRC & - & + & - \\
\hline Megan & $\mathrm{F}$ & 23 & Riwanda & - & - & - \\
\hline Kyewunyo & $\mathrm{F}$ & 5 & Uganda & - & + & - \\
\hline Peace & $\mathrm{F}$ & 21 & Uganda & - & - & - \\
\hline Connie & $\mathrm{F}$ & 18 & Uganda & - & - & - \\
\hline Bahati & $\mathrm{F}$ & 17 & Uganda & - & + & - \\
\hline Natasha & $\mathrm{F}$ & 17 & Uganda & - & - & - \\
\hline Becky & $\mathrm{F}$ & 16 & Uganda & - & - & - \\
\hline Sally & $\mathrm{F}$ & 16 & Uganda & - & - & - \\
\hline Cindy & $\mathrm{F}$ & 14 & Uganda & - & - & - \\
\hline Nkumwa & $\mathrm{F}$ & 11 & Uganda & + & - & - \\
\hline Kazahukire & $\mathrm{F}$ & 8 & Uganda & - & - & - \\
\hline Tumbo & $M$ & 18 & Uganda & - & + & - \\
\hline Mika & $M$ & 15 & Uganda & - & - & - \\
\hline Bwambale & $M$ & 7 & Uganda & - & - & + \\
\hline Rutoto & $M$ & 3 & Uganda & + & - & - \\
\hline Nagoti & $\mathrm{F}$ & 21 & Unknown & + & - & - \\
\hline Nani & $\mathrm{F}$ & 6 & Unknown & + & - & - \\
\hline Sum of PCR-positive samples & & & & 14 & 5 & 2 \\
\hline
\end{tabular}

DRC, Democratic Republic of Congo. 
Figure 1 Phylogenetic analysis of the novel chimpanzee rhadinovirus. A phylogenetic tree was constructed using the amino acid (aa) sequences encoded by the gB-DPOL segments of the novel primate herpesviruses and of known human and non-human primate herpesviruses, available in GenBank. Abbreviations of common names are used, and those of species names according to the ICTV (International Committee on the Taxonomy of Viruses) are given in parentheses. A multiple alignment of concatenated 1100 aa was analyzed with the neighbor-joining method. A rooted phylogram is shown, with CCMV as outgroup. The branch length is proportional to evolutionary distance (scale bar). Results of bootstrap analysis (1000 replicates) are indicated at the nodes of the tree. The novel PtroRHV-3 is highlighted in bold type. Full names of known viruses and their nucleotide sequence accession numbers are listed in the Materials and methods section.

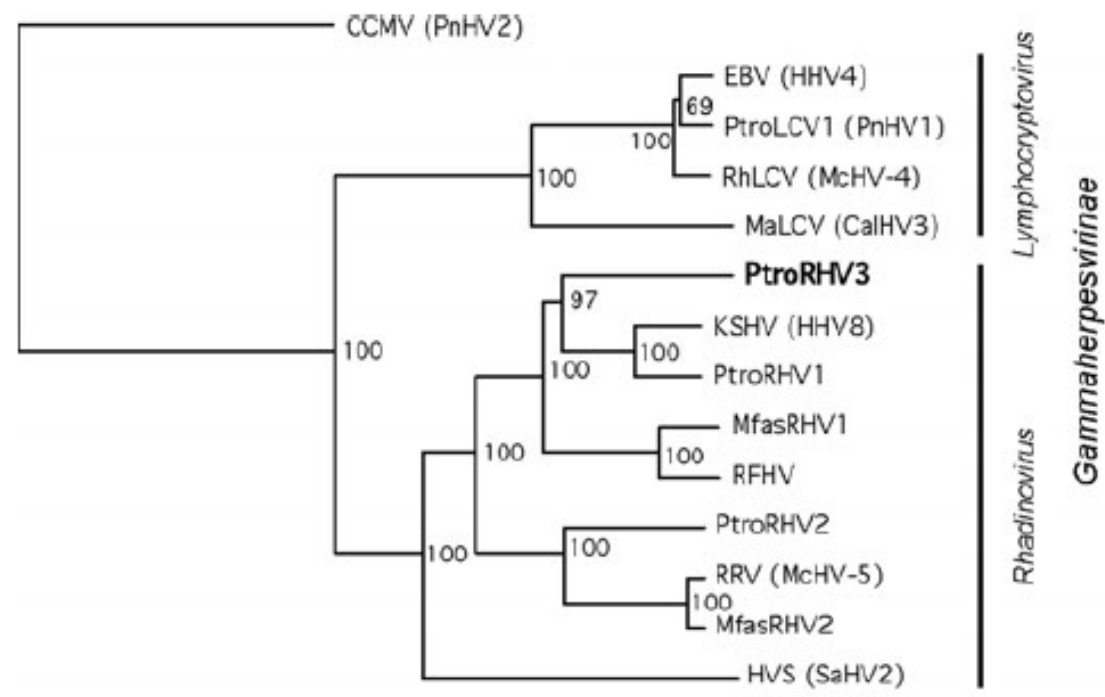

